\title{
Differential influences of negative emotion on spatial and verbal working memory: evidence from event-related potential and source current density analysis
}

\author{
Xuebing $\mathrm{Li}^{\mathrm{a}, \mathrm{c}}$, Xinying $\mathrm{Li}^{\mathrm{a}}$ and Yue-Jia Luo $\mathrm{o}^{\mathrm{a}, \mathrm{b}}$ \\ ${ }^{a}$ Key laboratory of Mental Health, Chinese Academy of Sciences, 'b State Key Laboratory of Cognitive Neuroscience and Learning, Beijing Normal University \\ and ${ }^{\mathrm{C}}$ Graduate School of the Chinese Academy of Sciences, Beijing, P.R. China \\ Correspondence and requests for reprints to DrYue-Jia Luo, MD, PhD, 19 Xin-wai St., Beijing 100875, China \\ Tel/fax: + 86105880 2365; e-mail: luoyj@bnu.edu.cn
}

Sponsorship: This work was supported by NSF China (30325026), Ministry of Education (106025) and the Chinese Academy of Sciences (KSCX2-SW-22I).

Received 30 May 2006; accepted 22 June 2006

We used the event-related potential technique to assess the influences of negative emotion on spatial and verbal working memory with a modified $n$-back task. In both spatial and verbal tasks, the anterior late positive component and the posterior P300 were affected by negative emotion. These effects were, however, greater in spatial tasks than in verbal ones. Especially, the parietal P300 was reduced by negative emotion in spatial tasks, but not in verbal tasks. Current density analysis revealed high current density in the frontal-parietal cortex, but only in the spatial tasks. The results suggest differential effects of negative emotion on spatial and verbal working memory and that the frontal-parietal cortex may be the area where negative emotion and spatial working memory interact. NeuroReport 17:1555-1559 (c) 2006 Lippincott Williams \& Wilkins.

Keywords: event-related potential, frontal-parietal cortex, negative emotion, source current density analysis, working memory

\section{Introduction}

It is well known that affect can modulate cognition. For example, there is considerable evidence that the influence of anxiety on performance may be mediated by effects on working memory, [1]. Working memory refers to a system used for the temporary storage and manipulation of information, which is deemed necessary for a range of more complex cognitive activities. In Baddeley's [2] model of working memory, spatial working memory is distinguished from verbal working memory, involving manipulation and retention of visuospatial and verbal information, respectively. Recent studies have shown that induced anxiety in normal subjects selectively impairs spatial, but not verbal, $n$-back task performance [3,4]. This implies that the modulation of cognition by affect is not a global effect but may involve specific neurocognitive mechanisms. It is, however, not known which neural mechanisms are involved in this selective modulation.

In the present study, we used the high temporal resolution event-related potential (ERP) technique to examine aspects of spatial and verbal working memory processing in negative and neutral emotional states. We also investigated, using source current density analysis, the potential cortical areas involved in the emotion-cognition interaction in working memory. In classical ERP studies with the $n$-back paradigm, frontal late positive component
(LPC) and parietal P300 elicited by memory items were regarded as working memory specific ERP components. Their amplitudes were directly related to working memory load in both spatial and verbal working memory tasks $[5,6]$. On the basis of these previous studies, we employed a modified 2-back task with aversive pictures before each trial, to induce negative emotional states while participants were performing working memory tasks. Neutral pictures were used as a control condition. Given that negative emotion selectively impairs spatial, but not verbal, working memory, we predicted that the frontal LPC and parietal P300 should be modulated by a negative emotional state only in spatial working memory tasks.

\section{Methods}

\section{Participants}

Fifteen volunteers (seven women) participated, aged 19-27 years $(M=21.7)$. All were right-handed with normal or corrected-to-normal vision and had no prior neurological/ psychiatric history.

Stimuli and procedure

Eighty-four digitized color pictures were selected from the International Affective Picture System [7]; 42 were classified as aversive pictures (e.g. mutilations) and 42 were classified 
as neutral pictures (e.g. household appliances). When presented, all pictures occupied between 3 and $4.5^{\circ}$ of visual angle on either side of the visual midline. Each target stimulus was drawn from a set of 12 English capital letters. The letter on a given trial appeared at one of 12 positions, each of which was on one of six equidistant radii of an imaginary circular array 2 or $6 \mathrm{~cm}$ from the screen's center.

Participants were seated in an electrically isolated, soundand light-attenuated room and viewed a computer monitor from a distance of $75 \mathrm{~cm}$.

All trials began with a picture, which remained on the screen for $1000 \mathrm{~ms}$, followed by a short interval (varied randomly from 200 to $400 \mathrm{~ms}$ ). Then a capital letter, serving as a target stimulus, was presented for $300 \mathrm{~ms}$. Participants were asked to decide whether the letter was the same as or different from that displayed two trials previously (hence, 2-back task). The stimulus sets of verbal and spatial working memory tasks were equivalent, but different instructions were given to the participants before the beginning of each task. In the verbal task, participants were instructed to remember the name of the letter and ignore its location. In the spatial task, the location of the letter was to be remembered. Half of them were told to press the ' $v$ ' key with their left index finger for the same stimulus and the ' $\mathrm{m}$ ' key with their right index finger for a different stimulus. For the other participants, the assignment of the response hand was reversed. The same and different stimuli were presented in equal proportions. If no response was made, the next trial would begin after $1500 \mathrm{~ms}$. Participants were informed that the pictures had nothing to do with their tasks, but they were requested to look at the pictures when they were presented.

To ensure that the induced emotion was stable during a period of time, the experiment was divided into two sessions. In one session, only the aversive pictures were presented, and in the other only neutral pictures were presented. Each session was composed of one verbal task and one spatial task. Each task contained four blocks and each block had 37 trials, resulting in 148 trials in each experimental condition.

Before the beginning of the experiment, participants were given a positive and negative affect schedule (PANAS), consisting of 10 positive affects (interested, excited, strong, enthusiastic, proud, alert, inspired, determined, attentive, and active) and 10 negative affects (distressed, upset, guilty, scared, hostile, irritable, ashamed, nervous, jittery, and afraid) [8]. Participants were asked to rate items on a scale from 1 to 5 ( $1=$ not at all, $5=$ very much), on the basis of the strength of emotion they were feeling at that moment. After each session of the experiment, participants filled out another PANAS scale.

\section{Event-related potential recordings and data analysis}

The electroencephalogram (EEG) was recorded from 64 scalp sites using $\mathrm{Ag} / \mathrm{AgCl}$ electrodes mounted in an elastic cap (NeuroScan Inc., Herndon, Virginia, USA), with the reference on the left and right mastoids. Vertical electrooculogram (EOG) recording electrodes were positioned above and below the left eyes, and horizontal EOG recording electrodes were positioned at the outer canthi of both eyes. All electrode impedances were kept below $5 \mathrm{k} \Omega$. The EEG and EOG were sampled at a digitization rate of $500 \mathrm{~Hz}$ and were band-pass filtered between 0.05 and $100 \mathrm{~Hz}$.
The EEG and EOG were digitally filtered offline with a $16 \mathrm{~Hz}$ low-pass filter, and were epoched into periods of $1200 \mathrm{~ms}$, from $200 \mathrm{~ms}$ before the onset of target stimuli to $1000 \mathrm{~ms}$ after target stimuli. Trials with artifacts were rejected with a criterion of $\pm 100 \mu \mathrm{V}$. ERPs were averaged for trials with correct responses.

The following 24 sites were chosen for statistical analysis: FPz, Fz, FCz, AF3, AF4, F1, F2, F5, F6, FC3, FC4, FT7 and FT8 (13 sites for anterior); Pz, POz, Oz, P1, P2, P5, P6, PO3, PO4, TP7 and TP8 (11 sites for posterior). The slow wave component may have separable anterior and posterior subcomponents. Thus, the anterior LPC and posterior LPC (labeled the P300 in this study) were measured separately in the $300-600$ and $280-430 \mathrm{~ms}$ time windows, respectively. Repeated-measures analysis of variance (ANOVA) was conducted on each ERP component with three factors: emotional state (negative/neutral), task type (spatial/verbal) and electrode site. Greenhouse-Geisser correction was used when the data violated the assumption of sphericity.

\section{Event-related potential source analysis}

Source analysis was performed on the negative emotion minus neutral emotion difference wave, using Curry V4.5 software (Neurosoft Inc., Herndon, Virginia, USA). After the grand-averaged ERPs were obtained, the standard magnetic resonance imaging head image supplied by the Curry software and the fiducial landmarks (the left and right preauricular points and the nasion) identified on it were fed into Curry. A computer algorithm was automatically performed in Curry to calculate the best-fit sphere encompassed by the array of electrode sites and to determine their spherical coordinates. The spherical coordinates for each recording site were used for the ERP current density analysis.

In the present study, we tentatively reconstructed the distributed sources over the time range $280-430 \mathrm{~ms}$ in a three-shell head model using the low-resolution electromagnetic tomography method.

\section{Results}

\section{Self-reported data}

The pictures induced the expected emotional states, on the basis of the self-reported changes in emotion. Paired $t$-tests between baseline and postsession PANAS scores were conducted on positive and negative PANAS subschedules in all participants. After the aversive experimental session, the negative PANAS scores increased $(t(14)=3.66, P=0.03)$ and positive PANAS scores decreased significantly $(t(14)=3.63, P=0.03)$. On the other hand, there were no overt changes in subschedule scores after the neutral experimental session.

\section{Behavioral data}

ANOVAs were conducted on response times and accuracy with two factors, emotional state and task type. No significant main or interactive effects on response times and accuracy were seen.

\section{Event-related potentials}

The anterior late positive component

A three-way ANOVA on LPC amplitudes revealed significant main effects of emotional state $[F(1,14)=30.16$, 

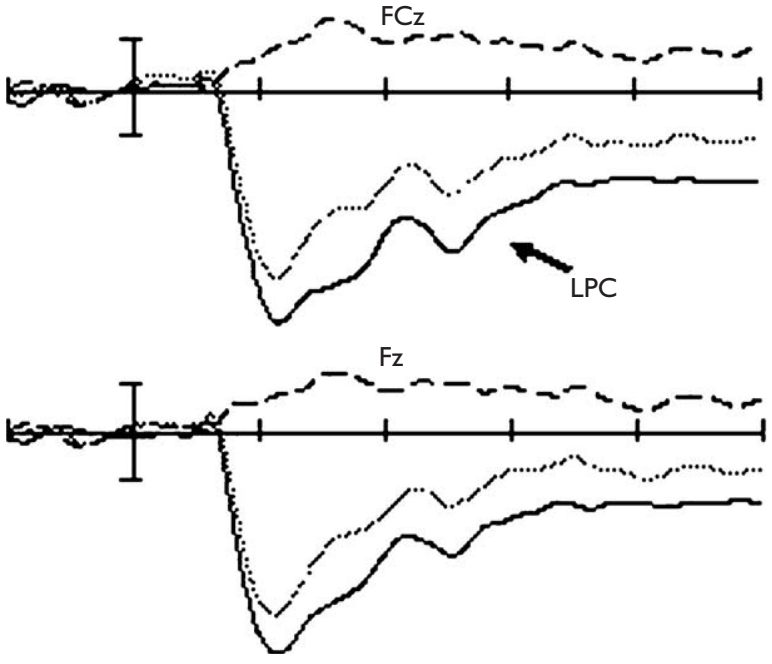

patial working memory

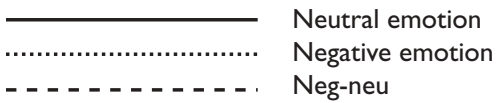

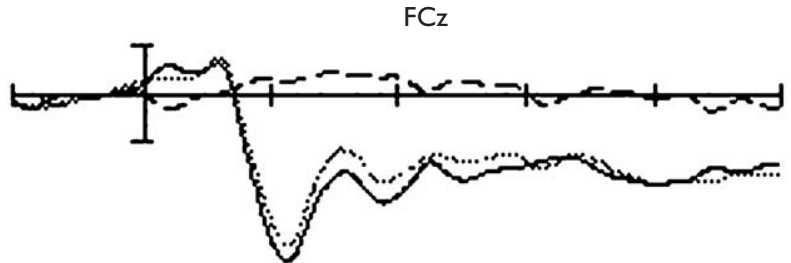
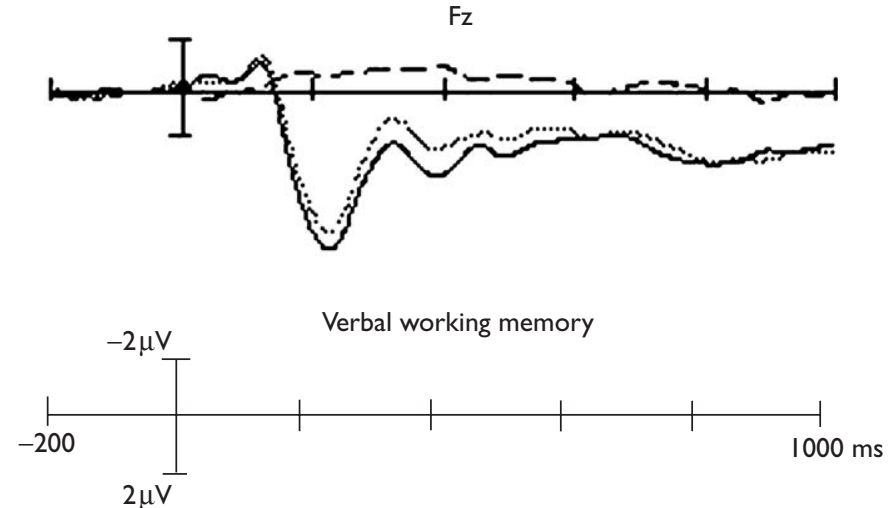

Fig. I Grand-averaged event-related potentials for targets in negative and neutral emotion as well as the difference wave (negative minus neutral) at Fz and FCz. Waveforms of spatial and verbal working memory are presented separately.

$P=0.0001]$ and task type $[\mathrm{F}(1,14)=7.3, P=0.02]$ (see Fig. 1 ). More importantly, there was an overt task type $\times$ emotional state interaction $[F(1,14)=25.6, P=0.0001]$. Further analysis showed that the LPC of negative emotion was smaller than that of neutral emotion in both spatial tasks $[\mathrm{F}(1,14)=44.7$, $P=0.0001]$ and verbal tasks $[\mathrm{F}(1,14)=7.2, P=0.018]$, and spatial tasks elicited larger LPC than verbal tasks did only for neutral emotion $[\mathrm{F}(1,14)=18.7, P=0.001]$. To demonstrate this interaction more clearly, difference waves were obtained by subtracting the averaged ERPs of neutral trials from those of negative trials (see Fig. 1). Repeated-measures ANOVA revealed that the difference waves' mean amplitudes between 300 and $600 \mathrm{~ms}$ were larger for spatial tasks than for verbal tasks $[\mathrm{F}(1,14)=17.1, P=0.001]$.

\section{The posterior $P 300$}

A three-way ANOVA on P300 amplitudes revealed a task type $\times$ emotional state interaction $[\mathrm{F}(1,14)=20.15, P=0.001]$. Further analysis showed that in the spatial task, P300 amplitudes in the negative emotion condition were smaller than those in the neutral emotion condition $[\mathrm{F}(1,14)=16.34$, $P=0.001]$. In contrast, in the verbal task, P300 amplitudes of negative and neutral emotion conditions were nearly the same $[F(1,14)=0.009, P=0.93]$ (see Fig. 2). In addition, there was a main effect of electrode site $[F(10,140)=26.69$, $P<0.001]$. Further analysis indicated that the largest amplitude was recorded on the Pz electrode.

\section{Event-related potential source analysis}

The reconstructed distributed sources overlaid onto the cortex of the standard brain model showed high current density in the frontal-parietal and temporal cortex in spatial tasks. In verbal tasks, only the temporal cortex was activated in the current density mapping (see Fig. 3).

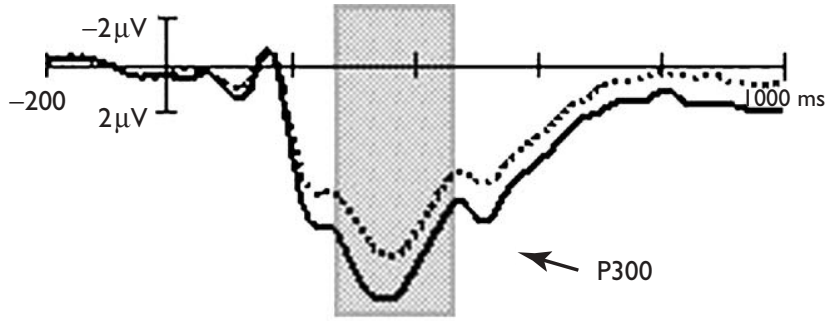

Spatial working memory

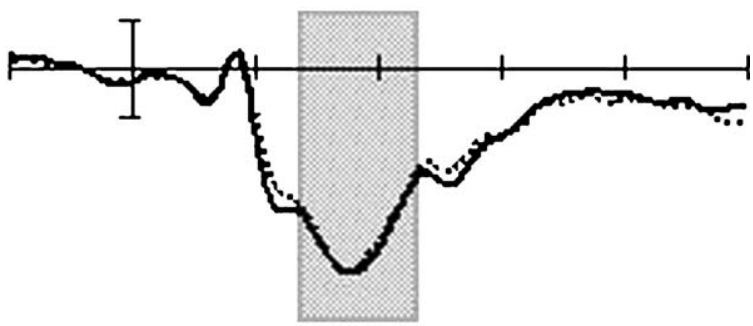

Verbal working memory

- Neutral emotion

...... Negative emotion

Fig. 2 Grand-averaged event-related potentials for targets in negative and neutral emotion at $\mathrm{Pz}$ in spatial and verbal working memory.

\section{Discussion}

The ERP results confirmed our prediction of differential influences of negative emotion on ERP correlates of spatial and verbal working memory. On the one hand, the degree to which frontal LPC was affected by negative emotion was different in the two types of tasks. On the other hand, the negative emotion selectively decreased parietal P300 of spatial tasks. 

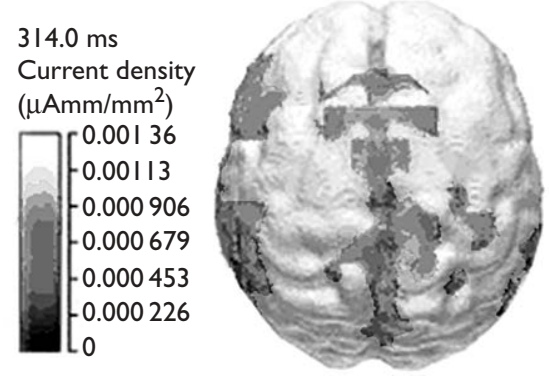

Spatial working memory

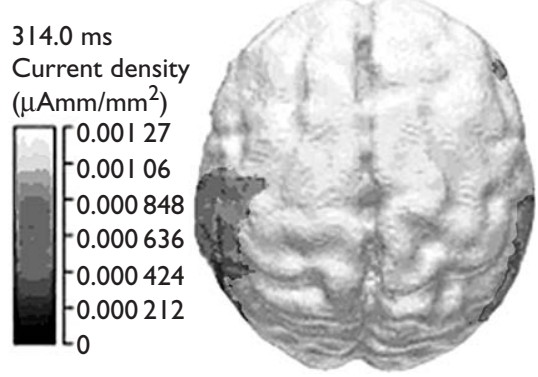

Verbal working memory

Fig. 3 The averaged current density distribution of the difference wave (Neg-neu) in spatial and verbal working memory at the time of difference wave peak (314 ms).

On the basis of the self-reported data, the experimental procedure induced a relatively high level of negative emotion. It is well known that aversive information has the ability to draw attention automatically [9], and the attentional resources taken up by negative emotion would no longer be available for other cognitive processes [1]. Hence, the attentional resource allocated to a task would be reduced. Frontal LPC amplitudes of both working memory tasks were attenuated significantly in the condition of negative emotion, which suggests that attentional resources for ongoing tasks were reduced.

From both the anterior LPC and posterior P300, we clearly observed that spatial working memory was affected by negative emotion more than was verbal working memory. In particular, reduced P300 amplitudes under negative emotion were only seen in the spatial task. These results can be interpreted in terms of the reliance of negative emotion and spatial working memory on a common visuospatial attention mechanism. Firstly, as discussed above, the induced negative emotion in the present study may automatically draw partial attentional resources away from tasks themselves. Secondly, the attentional resources are distinctly demanded in spatial working memory, but not necessarily demanded in verbal working memory. Previous studies have suggested that the rehearsal of locations in spatial working memory tasks is mediated by covert shifts of attention from one location to the next [10]. In contrast, the rehearsal of letters in verbal working memory tasks is based primarily on phonological processes [11]. Finally, both LPC and P300 are sensitive to the attentional demands of working memory tasks. Their amplitudes are larger when attention is exclusively focused on analyzing the eliciting stimulus, and smaller when attention is consumed by some other mental activity $[6,12]$. On the basis of these three points, it is possible that negative emotion and spatial working memory compete for attentional resources, so that the influence of negative emotion on the LPC and P300 was greater for the spatial than for the verbal task.

The results of the current density analysis provided further information on the cortical localization of these effects. We found that frontal-parietal regions were more active under negative emotion for spatial trials than for verbal trials. Neuroimaging studies have demonstrated that the superior frontal and parietal cortices are critically involved in the control of visuospatial attention $[13,14]$. Activity of these regions reflects increased frontal-parietal network engagement with increased task-related efforts or working memory demands [15]. Therefore, we assumed that the process of negative emotion limited visuospatial attention resources during the demanding spatial working memory task. Participants had to make greater efforts to accomplish the task. Hence, frontal-parietal regions were activated more.

\section{Conclusion}

We have provided the initial electrophysiological evidence for different effects of negative emotion on spatial and verbal working memory. Spatial working memory was more vulnerable to negative emotion, which was reflected by frontal LPC and parietal P300. In current density mappings, the difference between two types of tasks under negative emotion mainly involved activation of the frontal-parietal cortex, which is known to be a source of top-down control for spatial attention. These findings suggest that these cortical areas may play a key role in the interaction of spatial working memory and negative emotion.

\section{Acknowledgement}

We thank Professor Raja Parasuraman for his editorial assistance.

\section{References}

1. Eysenck MW, Calvo MG. Anxiety and performance: the processing efficiency theory. Cogn Emotion 1992; 6:409-434.

2. Baddeley A. The fractionation of working memory. Proc Nat Acad Sci 1998; 93:13468-13472.

3. Lavric A, Rippon G, Gray JR. Threat-evoked anxiety disrupts spatial working memory performance: an attentional account. Cogn Ther Res 2003; 27:498-504.

4. Shackman AJ, Sarinopoulos I, Maxwell JS, Pizzagalli DA, Lavric A, Davidson RJ. Anxiety selectively disrupts visuospatial working memory. Emotion 2006; 6:40-61.

5. Kusak G, Grune K, Hagendorf H, Metz AM. Updating of working memory in a running memory task: an event-related potential study. Int J Psychophysiol 2000; 39:51-65.

6. Gevins A, Smith ME, Le J, Leong H, Bennett J, Martin N, et al. High resolution evoked potential imaging of the cortical dynamics of human working memory. Electroencephalogr Clin Neurophysiol 1996; 98:327-348.

7. Lang PL, Bradley MM, Cuthbert BN. International affective picture system: technical manual and affective ratings. NIMH Center for the Study of Emotion and Attention; 2001 
8. Watson D, Clark LA, Tellegen A. Development and validation of brief measures of positive and negative affect: the PANAS scales. J Pers Soc Psycho 1988; 54:1063-1070.

9. Li X, Li X, Luo Y-J. Anxiety and attentional bias for threat: an eventrelated potential study. Neuroreport 2005; 16:1501-1505.

10. Postle BR, Awh E, Jonides J, Smith EE, D'Esposito M. The where and how of attention-based rehearsal in spatial working memory. Cogn Brain Res 2004; 20:194-205.

11. Sakai K, Passingham RE. Prefrontal selection and medial temporal lobe reactivation in retrieval of short-term verbal information. Cereb Cortex 2004; 14:914-921.
12. McEvoy LK, Smith ME, Gevins A. Dynamic cortical networks of verbal and spatial working memory: effects of memory load and task practice. Cereb Cortex 1998; 8:563-574

13. Corbetta M, Miezin FM, Shulman GL, Petersen SE. A PET study of visuospatial attention. J Neurosci 1993; 13:1202-1226.

14. Yantis S, Schwarzbach J, Serences JT, Carlson RL, Steinmetz MA, Pekar JJ, et al. Transient neural activity in human parietal cortex during spatial attention shifts. Nat Neurosci 2002; 5:995-1002.

15. Rama P, Kesseli K, Reinikainen K, Kekoni J, Hamalainen H, Carlson S. Visuospatial mnemonic load modulates event-related slow potentials. Neuroreport 1997; 8:871-876. 